\title{
Cavity preparation using an Erbium:YAG laser
}

\author{
A clinical evaluation of an Erbium:YAG laser for dental cavity preparation by D. J. P. Evans, S. Matthews, N. B. Pitts, \\ C. Longbottom, and Z. J. Nugent Br Dent J 2000; 188: 677-679
}

\begin{abstract}
Objective
A randomised controlled trial to determine the acceptability to dentists and patients of cavity preparation with an Erbium:YAG laser as compared with conventional handpieces.

Methods

Fifteen dentists (9 GDPs, 1 community dentist and 5 hospital dentists) treated 77 patients (age range 3.5-68 years old) who had two matched cavities, in a split mouth, randomised trial. One cavity was prepared conventionally, the other with the laser, with dentist and patient preference determined by questionnaire.
\end{abstract}

\section{Results}

In the majority of cases, where dentists expressed a preference, it was for conventional cavity preparation, and this was significant $(P<0.001)$. In more than half of the laser appointments, dentists had to use conventional handpieces to complete the cavity. Principle difficulties reported with the laser were access ( 25 cases) and slow speed of cutting ( 11 cases). Patients aged $\geq 10$ years who expressed a preference, preferred laser treatment, and this was significant $(P<0.001)$. Patients aged $<10$ years, assessed using a simplified pictorial questionnaire, did not show a significant preference for either technique.

\section{Conclusions \\ Dentists preferred conventional handpieces for cavity preparation while patients aged $\geq 10$ years old preferred laser treatment. Patients $<10$ years old did not express a preference.}

\section{In Brief}

- This study found that dentists preferred conventional methods of cavity preparation to using an Erbium:YAG laser.

- Patients preferred laser cavity preparation, unless they were younger than 10-years-old, when they did not show a preference for either method.

- The range of difficulties reported by dentists when using the laser indicate that, at the current stage of development, lasers are not a realistic alternative to conventional methods of cavity preparation.

\section{Comment}

$\mathrm{M}$ any of the initial clinical studies of lasers in the mouth were concerned with soft tissue surgery, and it was realised from an early stage that the $\mathrm{CO}_{2}$ laser was an effective means of excising oral soft tissue lesions. ${ }^{1-3}$ However, the development of a system with suitable wavelength and delivery mechanism to enable cutting of enamel and dentine without damaging the pulp has been much more problematic.

The Erbium:YAG system has recently become available, and early clinical studies appear promising. It emits infra-red radiation which is absorbed by water and hydroxyapatite, and is intended for application on hard tissues. This is the first dental laser for use in cutting human teeth in vivo and was cleared by the US Food and Drug Administration for marketing in USA. However, alongside the article by Cosean et al., ${ }^{4}$ the American Dental Association expressed concerns about its clinical usefulness, especially in posterior teeth, for caries removal and cavity preparation; the rapid temperature rises and potential damage to hard tissues, the dental pulp and periodontium; and the limited peer-reviewed studies on the safety and effectiveness of the system.

This paper by Evans et al. is therefore extremely timely and relevant. It presents a randomised controlled trial to determine the acceptability of an Erbium:YAG laser as compared with conventional handpieces. The comparative element in this study is important, because dentists wish to know how a new technique measures up to the conventional standard procedure. This rigorous control has usually not been a feature of the multitude of papers published on lasers in dentistry, where patients have often been invited to 'try' the new dental laser ${ }^{5}$ without reference to conventional techniques.

The results are interesting in that in the majority of cases, the dentists preferred conventional drills for cavity preparation, mainly because of difficulties with access and slow speed of cutting. However, patients over the age of 10 years preferred the laser, probably because it was perceived as being less painful and having less vibration than the drill, while patients less than 10-years-old did not express a preference. Clinical studies using this design are an important means of evaluation for new dental lasers.

1 Tuffin J R, Carruth J A S. The carbon dioxide surgical laser. Br Dent J 1980; 149: 255-258.

2 Frame J W. Treatment of sublingual keratosis with the $\mathrm{CO}_{2}$ laser. $\mathrm{Br}$ Dent $J$ 1984; 156: 243-246.

3 Frame J W. Removal or oral soft tissue pathology with $\mathrm{CO}_{2}$ laser. J Oral Maxillofac Surg 1985; 4: 850-855.

4 Cozean C, Arcoria C J, Pelagalli J, Powell L G. Dentistry for the 21st Century? Erbium: YAG laser for teeth. J Am Dent Assoc 1997; 128: 10801087.

5 Baggett F J, Mackie I C, Blinkhorn A S. The clinical use of the Nd: YAG laser in paediatric dentistry for the removal of oral soft tissue. $\mathrm{Br}$ Dent J 1999; 187: 528-530.

\section{J. W. Frame}

Professor of Oral Surgery, University of Birmingham, President-elect, International Society for Lasers in Dentistry ISLD 